\title{
A SURVEY ON BANDWIDTH ALLOCATION SCHEMES IN WSNS USING TDMA BASED MAC PROTOCOL
}

\author{
Ratna B. Patil ${ }^{1}$, S. K. Padaganur ${ }^{2}$, J. D. Mallapur ${ }^{3}$, Namrata Bannur ${ }^{4}$ \\ ${ }^{1}$ M. Tech (DCN) Student, Department of ECE, B.L.D.E.A's Dr. P.G.Halakatti CET Vijayapur, Karnataka, India. \\ ratnapatil92@gmail.com \\ ${ }^{2}$ Assistant Professor, Department of ECE, B.L.D.E.A's Dr. P.G.Halakatti CET Vijayapur, Karnataka, India. \\ padaganursk@gmail.com \\ ${ }^{3}$ Professor, ECE Department of BEC, Bagalkot, Karnataka, India \\ bdmallapur@yahoo.co.in \\ ${ }^{4}$ M. Tech (DCN) Student, Department of ECE, B.L.D.E.A's Dr. P.G.Halakatti CET, Vijayapur, Karnataka, India. \\ namratabannur1992@gmail.com
}

\begin{abstract}
Wireless sensors networks play an important role in wireless communication due to its various advantageous characteristics and applications. The real time applications of WSNs such as bandwidth sensitivity and sharing priority especially their capacity of artificial intelligence between sensor node communications are very important factors. The limited battery power of nodes, lesser storage capacity, processing speed, collision between the nodes, overhearing, idle listening and limited bandwidth allocation are the major constraints of wireless sensor networks. There are many approaches to meet these actual requirements of WSNs. In this paper we study designing of MAC layer by considering the bandwidth in terms of time slot allocation in a pure TDMA/CSMA based networks, which provides considerable amount of profit by avoiding collision, overhearing idle listening and finally provides energy efficiency. Bandwidth allocation can be studied by considering the different types of network topologies.
\end{abstract}

Keywords: DSN, WSN, MAC, TDMA.

\section{INTRODUCTION}

A wireless sensor network (WSN) is also known as a wireless sensor and actuator network (WSAN). These networks are defined as the group of large number of tiny, autonomous, low power, low cost and multifunctional sensor nodes deployed in the environment depending upon the requirement. We can see the sensors as depicted in Fig 1.

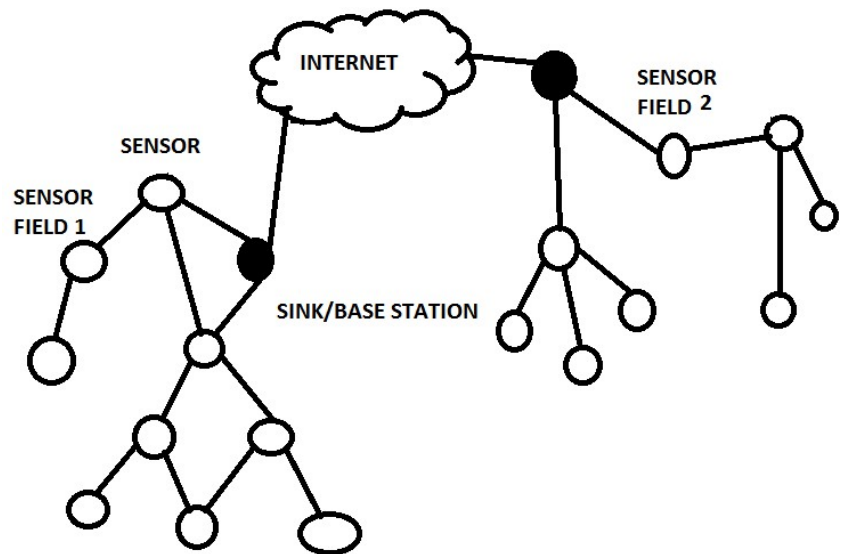

Fig 1. WSN nodes

The WSNs can be popularly classified as terrestrial, underground, aquatic, multimedia, and mobile communication fields depending upon the application such as defense, forest, medical science and industry in order to know the physical and environmental conditions, such as temperature, pressure, etc. We can see the architecture of sensor network in Fig 2. A wireless sensor network (WSN) consisting of sensor nodes, monitors the physical conditions of the environment where they are deployed and gather the physical environmental parameters to communicate with base station [1]. The sensor nodes are strongly deployed in regions where they guide many physical phenomena such as vibration, pressure, temperature, movement of objects, humidity, light conditions, radiations, and noise levels depending upon the location. The sensor nodes make selforganization in order to form an Ad-hoc network after the deployment.

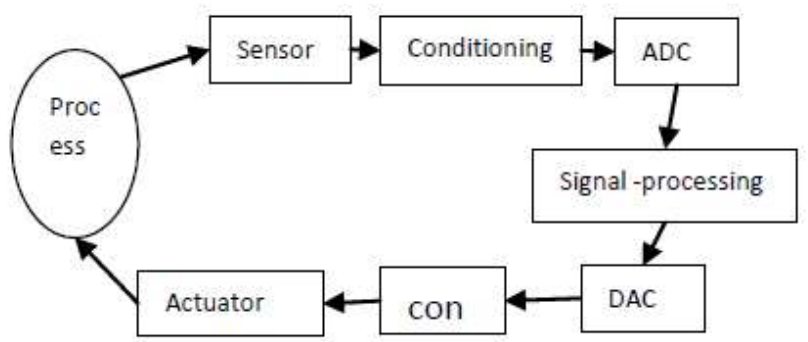

Fig 2. Architecture of WSN

Sensor nodes are resource constraint, as these are equipped with batteries with limited power, tiny microprocessors/ microcontrollers, low power transceivers, and sensors for 
gathering information about the deployed environment. Individual sensor nodes in wireless sensor networks are inherently unreliable and sensor will have very limited capabilities to reach the real time requirement and real-time guarantees. There is a new development in the research area of WSNs called Distributed or dispersed sensor networks (DSNs) [12] that has recently emerged as an important research area. This development has been spurred by advances in sensor technology and computer networking. It is economically feasible to implement DSNs, but there are several technical challenges that must be achieved. There after we can use them for gathering the day-today's information in increased complexity. We can see the block diagram of DSN as depicted in Fig.3. The ultimate goal of DSN or WSN is to provide more reliable services and to avoid end-to-end delays, with effective allocation of bandwidth and least consumption of energy by the sensors in the underlying Sensor network.

The primary goal of sensor networks is to make decisions or acquire knowledge based on the information fused from distributed sensor nodes. In the bottom, sensor node senses the data or information from different sensing events or from different conditions. Initially data processing is done by data aggregation, which can be carried out at the local node i.e. from sensor to cluster heads, to generate local event detection result. This resulting intermediate information will then be integrated or fused at an upper processing unit to derive knowledge and help making decisions.

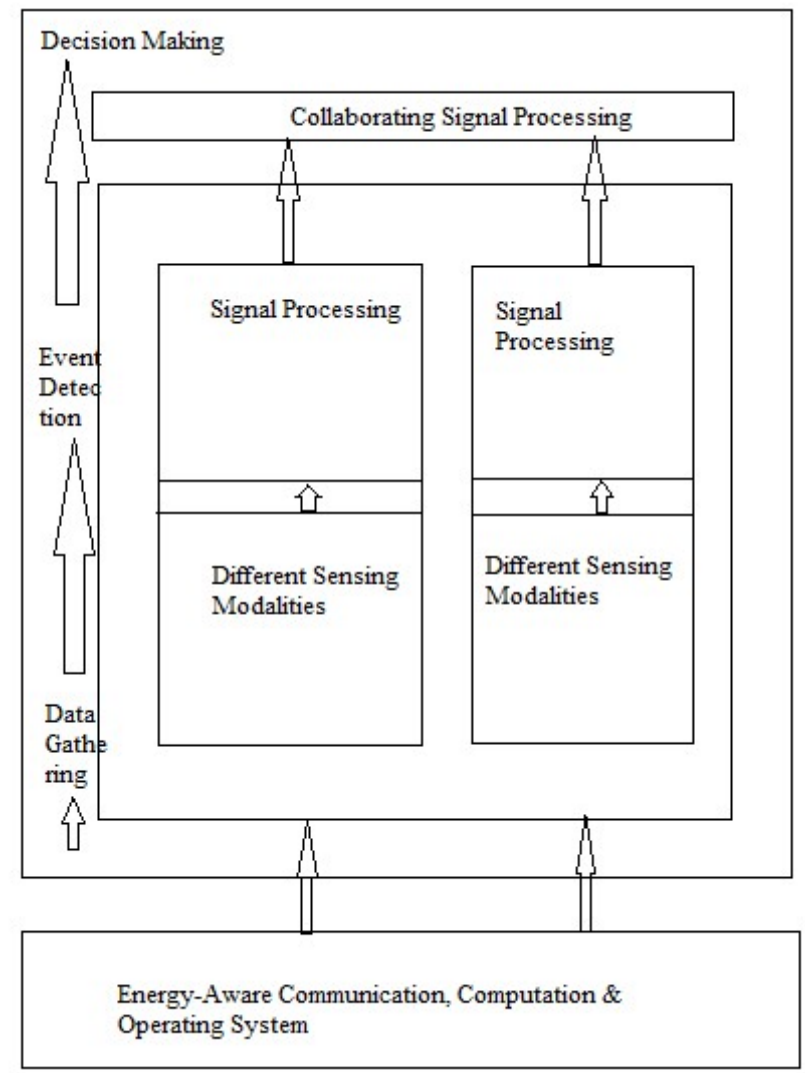

Fig 3. Block diagram of DSN
Cost of sensor nodes depends on the size of the sensor. If the sensors size is shorter, then price of nodes will be cheaper, so that more sensors can be deployed to achieve quality through quantity.

In WSN the sensed data from the each sensor nodes may take transmission in multiple paths to reach the destination. The total available channel bandwidth will be portioned, and the response time it takes is very large [5]. In the real time applications of WSNs, the wireless sensor networks are sensitive to allocated bandwidth and needs higher share of bandwidth for higher priority data to meet the dead line requirements. Higher Priority data should be allowed to use maximum share of bandwidth to achieve the deadline requirements. MAC layer gives assistance is that, the routing algorithm made in such a way that the multiple noninterleaving and prioritized paths must be made available to the routing table. In the following study, we discuss the MAC protocol that is based on TDMA method. With the use of TDMA, the MAC protocol guarantees to overcome the channel access delay binding the end-to-end transmission time of the network layer routing protocol.

In the following sections, we will study in detail MAC use in WSN and the factors to be considered for the effective performance of MAC protocol in section 2. In addition, we will discuss the major classification of MAC protocols in section 3; we describe general bandwidth allocation and scheduling scheme in WSN in section 4. And in section 5 we study the TDMA based MAC with its advantages and drawbacks And later in section 6 we conclude about the literature study about WSNs.

\section{MEDIUM ACCESS CONTROL IN WSN}

MAC is one of the major issues in designing of WSNs. MAC is used firstly, to avoid collision of data that is caused by the transmission of data packets over same medium when transmission is done between two or more sensor nodes. Second reason is to provide fair and efficient share of bandwidth resource among multiple sensor nodes. Hence, MAC plays a very important role in network operation and performance of WSNs. Some important parameters that must be considered while designing MAC protocol are energy efficiency: batteries power the sensor nodes and it is very difficult to recharge the batteries or reallocate the power to the senor nodes once they are deployed. Scalability and adaptability: a good MAC protocol should be adjustable to changes in sensor nodes. i.e capabable of providing service to newly updated sensor nodes along with deployed ones. Accommodate such changes gracefully, latency: refers to delay between the packet transmission from sender to receiver or source to destination. Throughput: is successful transmission of data from sender to receiver in a given time interval. Channel utilization: is the effective utilization of allocated band of frequency in a channel. In addition, fairness: the ability of sensor nodes to equally distribute or share the common transmission channel among themselves. 


\section{CLASSIFICATION OF MAC}

There are many types of MAC protocols but depending upon the requirement and application, we can categorize into contention-based MAC protocol and schedule-based MAC protocol

\section{I] Contention-based MAC protocol}

In contention-based MAC protocols, there will be common medium between all nodes for transmission of data or information. Thus, there may be a chance of occurrence of collision during the contention process. In order to avoid the collision, shared channel access can be arbitrated through some probabilistic coordination. Before transmission the sender listens to the shared medium, waits a random period. Suppose if the medium is found busy and then retries the transmission after some time. Therefore, the contention based MAC is better for networks having contention and burst traffic is expected.

The typical examples of contention-based MAC protocols are ALOHA (Additive Link On-line Hawaii System) and CSMA (Carrier Sense Multiple Access).

\section{II] Schedule-based MAC Protocols}

In schedule-based MAC protocol, shared medium used for transmission is divided in respect to either frequency (Frequency Division Multiple Access) or time (Time Division Multiple Access) or orthogonal pseudo - noise codes (Code Division Multiple Access). By this way, different nodes can access the shared medium without interfering with each other and thus effectively avoids collisions. Since most of the Schedule-based MAC protocols are TDMA-based they consume less energy i.e. more energy efficient as compared to Contention-based MAC protocols, because contention based protocols waste energy in collisions and idle listening in the network.

\section{BANDWIDTH ALLOCATION \\ SCHEDULING IN WSN}

AND

The various multiplexing techniques such as FDMA, TDMA, and CSMA used to overcome the bandwidth allocation problem in wired networks and cellular networks have been used in WSNs as well. However due to the limitations of energy and cost most of the implementations consider CSMA and TDMA based approaches. Type of network topology used also plays an important role in allocation of bandwidth due to interference associated with different types of topologies in WSNs.

WSNs rely on three different types of networks star, cluster tree and mesh. Due to least hopping property of star topology it is least complex, because a star topology will have central coordinator device and all the surrounding neighbors are with single hop distance so they can listen to everyone within network, there won't be any problems such as hidden node exposed or shortage [6] as shown in fig 6 . We will assume that a coordinator device has more energy capability than destination devices and direct end-to-end communication between end devices is not allowed. Moreover, the problem of bandwidth allocation for multichip topology networks been found NP-complete.
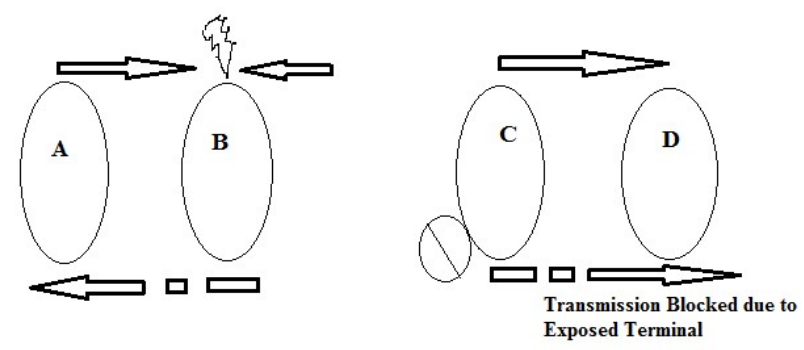

Fig 4. Hidden and Exposed terminal

\section{TDMA-BASED MAC PROTOCOL}

Among the critical issues in design of wireless sensor network, MAC plays the first role.

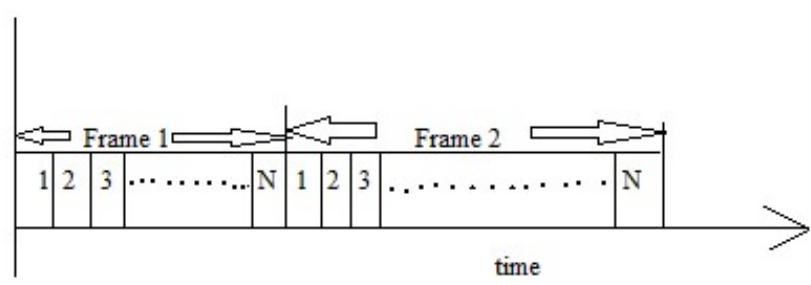

Fig 4. TDMA Frame Structure

In TDMA based MAC protocol, time is divided into different time frames which are further divided into fixed number of time slots we can see the frame structure of TDMA in Fig 4.

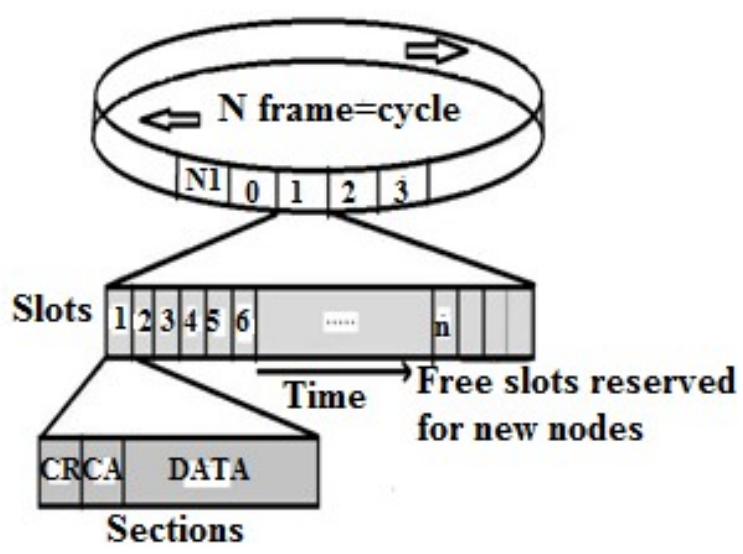

Fig 5. TDMA Based-MAC protocol

In TDMA each node is allocated with different time slots, different time-frame and each node is allowed to transmit only during the permitted time-slot. A node depending on schedules of neighboring node may remain in sleep mode when it is neither ready to transmit or nor to receive data packets, that is a node can switch off its transceiver operation and conserve the considerable amount of energy which makes the system energy efficient. 
In contention based channels due to common medium there may not be effective utilization of allocated bandwidth, due to the collision. There may be chances of retransmission of data packets that leads to loss utilization of more energy resource it can be a cause for energy constraint problem. As the TDMA is scheduling based, it provides delay guarantee, avoids collisions due to allocation of time slots with different time frames. Along with these advantages TDMA has some of the negative factors as well. It uses topology information, limited adaptability and scalability to changes in WSN nodes and to avoid the end-to-end delay time synchronization is needed.

\section{CONCLUSION}

The survey presents the detailed overview of WSNs with its advantages and associated constraints; we have studied use of MAC in WSN and the factors that have to be considered while designing the MAC protocols. In this paper we also described the major classifications of MAC protocol that are classified depending upon the requirement and application and the paper describes the approach of TDMA based MAC protocol with its overcomes in energy constraints of WSN such as bandwidth efficiency, collision, idle listening, overhearing etc as compared to the other multiplexing techniques such as CDMA, FDMA. The TDMA is better both in performance as well cost of deployment. We studied the bandwidth allocation dependency of WSNs with the considerations of different network topologies. We can conclude that along with these advantages characteristics, TDMA also has some of the negative factors. So in future study, the problem of synchronization TDMA should overcome to meet the real time requirements of WSNs.

\section{REFERENCES}

[1]. Priority based bandwidth allocation in Wireless sensor networks (IJCNC) vol.6, no.6 Nov 2014

[2]. Kim and p. S. Joon, "reliable and real time data dissemination in wireless sensor networks," inproc. Of IEEE MILCOM, pp.1-5, San Diego, ca, 2008.

[3]. Analysis of QoS in wireless sensor network (IJARCET) volume 4 issue 2, February 2015

[4]. bandwidth assignment in a cluster-based wireless sensor network WCE 2013, July 3 - 5, 2013, London, U.K.

[5]. T Qureshi and D.A.Zighed, "Proposition and Simulation of a Network Layer based Hard Real time

Protocol for Wireless Sensor Networks," in Proc. of IEEE Mobile and Wireless Communications

[6]. Summit, pp. 1-5, Budapest, Hungary, 2007.Fair end-toend bandwidth distribution in Wireless sensor networks IEEE ICC 2010 proceedings.

[7]. A Survey on TDMA-based MAC Protocols for Wireless Sensor Network Website: www.ijetae.com (ISSN 22502459, ISO 9001:2008 Certified Journal,

[8] A survey on Bandwidth resource Allocation and scheduling in wireless sensor Networks 2009 International Conference on Network-Based Information System. 